\section{Prevalencia y determinantes de adherencia a terapia antihipertensiva en pacientes de la Región Metropolitana}

\author{
PAOLA VARLETA ${ }^{1,2}$, CARLOS AKEL $^{1,3}$, MÓNICA ACEVEDO ${ }^{1,4}$, \\ CLAUDIA SALINAS $^{1, \mathrm{a}}$, JAVIER PINO $^{5, \mathrm{c}}$, VIVIANA OPAZO ${ }^{1, \mathrm{~b}}$, \\ ANA GARCÍA ${ }^{6}$, CAROLINA ECHEGOYEN ${ }^{7}$, \\ DANIEL RODRÍGUEZ ${ }^{8}$, LISSETTE GRAMUSSET ${ }^{9}$, \\ SANDRA LEÓN ${ }^{10}$, PEDRO COFRÉ ${ }^{11}$, HILDA HERNÁNDEZ ${ }^{12}$, \\ PATRICIA NEIRA $^{13}$, RAQUEL RETAMAL ${ }^{14}$, \\ GLORIA PETIT ${ }^{15}$, NATALIA MOYA ${ }^{16, d}$
}

\section{Assessment of adherence to antihypertensive therapy}

Background: Lack of adherence with medications is the main cause of antihypertensive treatment failure. Aim: To assess adherence to antihypertensive drugs and its determinants. Material and Methods: The Morinsky-Green questionnaire to determine treatment adherence was applied to 310 hypertensive patients from primary care centers, aged $60 \pm$ 10 years ( $65 \%$ females) in treatment for $4 \pm 1$ months. Socio-demographic features, use of medications and quality of life using EQ5D questionnaire were also assessed. Results: Twenty percent of patients were diabetic and $19 \%$ were smokers. Fifty four percent were adherent to therapy. A higher age and being unemployed were associated with a higher compliance. The main reasons to justify the lack of adherence were forgetting to take the pills in $67 \%$ and adverse effects in 10\%. Only diastolic pressure was lower in adherent patients, compared with their non-adherent counterparts (78 \pm 12 and $81 \pm 17 \mathrm{mmHg}$, respectively $p<0.01$ ). Conclusions: Only half of hypertensive patients comply with their antihypertensive therapy.

(Rev Med Chile 2015; 143: 569-576)

Key words: Antihypertensive Agents; Hypertension; Medication Adherence; Patient Compliance.

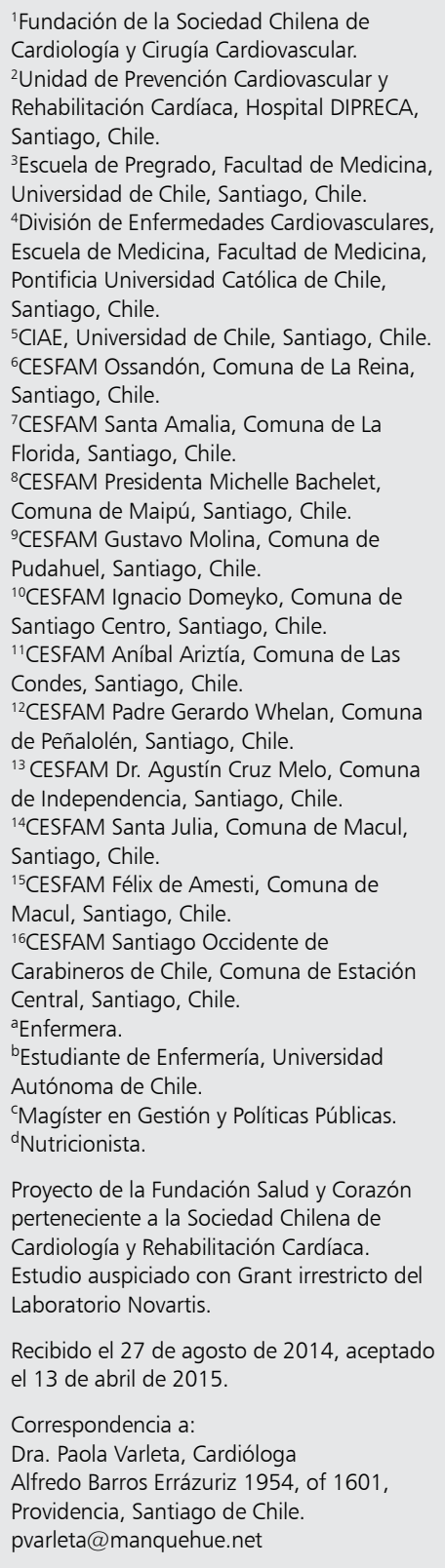

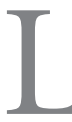

a hipertensión arterial (HTA) es un problema mayor de salud pública. Se le atribuye cerca de $54 \%$ de la enfermedad cerebrovascular y $47 \%$ de la enfermedad cardíaca isquémica ${ }^{1,2}$. Según la última Encuesta Nacional de Salud, la HTA es la principal enfermedad crónica declarada en la población, siendo su prevalencia de $26 \%{ }^{3}$. Del total de personas con presión arte- rial (PA) elevada en Chile, 37\% refiere estar con tratamiento farmacológico y sólo $16 \%$ tiene la PA controlada.

La falta de adherencia al tratamiento antihipertensivo (TrHTA) es una importante causa del pobre control de ésta. La mitad de los pacientes que inician un TrHTA, abandona por completo la atención dentro del primer año y de los que 
siguen en tratamiento, sólo la mitad adhiere ${ }^{4}$. En el Reino Unido, entre 40 y $50 \%$ de los pacientes que inician un nuevo TrHTA mantienen la medicación a seis meses ${ }^{5}$.

Se define adherencia al tratamiento crónico como el grado en que el comportamiento de una persona - tomar el medicamento, seguir un régimen alimentario y ejecutar cambios del modo de vida - se corresponde con las recomendaciones acordadas con un profesional del área médica. Estudios mencionan como factores asociados a una menor adherencia: la menor edad, bajo nivel educacional, deficiente apoyo familiar, y deficiente relación médico paciente ${ }^{6}$.

Para monitorear adherencia existen procedimientos directos, como medir metabolitos en la sangre e indirectos. Entre los indirectos, se incluyen la percepción del médico, conteo de medicamentos, y el autorreporte ${ }^{7}$. Dentro de las herramientas del autorreporte existen cuestionarios como el de Morisky-Green-Levine (MGL), fácil de implementar y validado en distintos países ${ }^{8-9-10}$.

En Chile disponemos de escasa información sobre adherencia al TrHTA. El propósito de este trabajo es determinar la adherencia al tratamiento farmacológico antihipertensivo en pacientes hipertensos de la Región Metropolitana e investigar factores asociados a la falta de adherencia.

\section{Pacientes y Método}

Estudio prospectivo en dos etapas: reclutamiento, y seguimiento con intervención. La data presentada corresponde a la etapa de reclutamiento con diseño de corte transversal. Se invitó a participar a sujetos hipertensos entre 25 y 80 años incorporados al programa cardiovascular de centros de atención de salud familiar (CESFAM) del Área Metropolitana. El diagnóstico de HTA se basó en flujograma No 1 para confirmación de diagnóstico de HTA de Guía Clínica MINSAL ${ }^{11}$. El médico investigador de cada CESFAM debía incorporar pacientes hipertensos autovalentes de su práctica cotidiana, con terapia farmacológica de $\geq 30$ días a $<6$ meses y sin criterios de exclusión (insuficiencia cardíaca, infarto miocárdico, ictus, insuficiencia renal en diálisis, enfermedad mental, analfabetos o con discapacidad física para leer o escribir). Se obtuvo muestra de 605 pacientes, los cuales fueron invitados a participar en el estudio por personal de la Fundación de la Sociedad Chi- lena de Cardiología y Cirugía Cardiovascular. Se incorporaron 310 (39 no contactados, 15 excluidos y 242 rechazaron participar) entre octubre de 2012 y agosto de 2013.

En la visita a todo paciente se le realizó toma de presión arterial (PA) con equipo automático Omron HEM-742, que promedia tres tomas, luego de reposo de 5 minutos. Cada sujeto respondió encuesta sobre antecedentes demográficos, escolaridad, factores de riesgo cardiovasculares, hábitos de alimentación, relación médico-paciente, características del TrHTA (efectos adversos, factores relacionados al cumplimiento), y autopercepción de salud, para lo cual se utilizó el cuestionario EQ5D, sin incorporar escala análoga visual ${ }^{12}$. Se consideró autopercepción de salud óptima el responder las 5 preguntas satisfactoriamente. Se aplicó el cuestionario de MGL para determinar adherencia farmacológica al TrHTA, considerándose adherente aquel sujeto que respondió adecuadamente a las 4 preguntas del cuestionario.

Para la evaluación de alimentación saludable se incorporaron 5 preguntas referentes a consumo de: frutas y verduras, salero, pan con o sin sal, embutidos y enlatados. Se confeccionó un score de hábito de alimentación saludable (SHA), obteniéndose un valor de 1 cuando se respondió adecuadamente a cada una de las preguntas, y de 0 con todas incorrectas, traduciendo una inadecuada adherencia alimenticia. Se estimó que el consumo en g de sal para un score de 1 era de $4 \mathrm{~g}$ (1.550 mg de sodio), y para score de 0 era de al menos $9 \mathrm{~g}$ al día ${ }^{13}$.

Con respecto a la relación médico paciente se consideró óptima si el paciente respondía positivamente a sentirse acogido en sus controles médicos, tener confianza de preguntar por posibles efectos indeseados, y de informar sobre no toma de fármacos.

\section{Análisis estadístico}

Se utilizó para el análisis estadístico software estadístico STATA. Los valores se presentan como media \pm DE para las variables continuas y como porcentaje para variables discretas. Para comparar grupos independientes, se utilizó test exacto de Fisher para variables categóricas y la prueba t de Student para variables continuas. Se realizó análisis de regresión logística considerando como variable dependiente la adherencia por MGL, con cada uno de las variables seleccionadas. Los estimadores beta de las regresiones logísticas son 
efectos marginales (EM) o elasticidades. El modelo de regresión logística general utilizado para el análisis fue el siguiente:

$$
\gamma=\beta_{0}+\beta_{1} X_{1}+\beta_{2} X_{2}+\beta_{3} X_{3}+\mu
$$

Donde Y es la variable dependiente dicotómica del test MGL; $X_{1}$ corresponde a variables sobre características personales tales como edad, estado civil, ocupación, nivel educacional; $X_{2}$ son variables independientes que reflejan el consumo de determinados grupos de medicamentos para el TrHTA y $X_{3}$ representa un conjunto de variables acerca de sus hábitos alimenticios, autovalencia y relación con su médico tratante. $\mu$ es el error estocástico de la regresión logística.

\section{Aspectos éticos}

El estudio se realizó bajo la aprobación del Comité de Ética Oriente del Área Metropolitana. Todos los pacientes firmaron consentimiento informado en la visita previa recolección de datos.

\section{Resultados}

Se incluyeron 310 pacientes de once CESFAM, 64,5\% mujeres, edad promedio $60 \pm 10$ años. La duración promedio del TrHTA fue de: $4 \pm 1$ meses ( 1 a 2 controles médicos). Todos los pacientes habían tenido consejería nutricional. Las características demográficas de la población y prevalencia de factores de riesgo se presentan en la Tabla 1.

La PA sistólica (PAS) y diastólica (PAD) promedio fue de $142,6 \pm 31,6 \mathrm{mmHg}$ y 79,6 $\pm 12,1$ $\mathrm{mmHg}$, respectivamente. Se observó en mujeres menor PAS $(\mathrm{p}<0,1)$ y PAD $(\mathrm{p}<0,05)$ que en hombres. En relación al TrHTA, el número de comprimidos promedio de la población estudiada fue de 2,1 comprimidos al día. La prescripción más frecuente fueron los bloqueadores del receptor de la angiotensina (48\%) e inhibidores de enzima convertidora de angiotensina (38\%), y en horario de administración matinal (97\%). El resto de la información se detalla en Tabla 2.

Tabla 1. Características demográficas y clínicas de la población estudiada total y por sexo (información expresada como porcentaje o promedio \pm DE)

\begin{tabular}{|lcccc|}
\hline & Hombres (n: 110) & Mujeres (n: 200) & Total (n: 310 ) & p \\
\hline Edad (años) & $59,9 \pm 10$ & $60,5 \pm 10,7$ & $60,27 \pm 10,4$ & NS \\
Estado civil & & & & \\
Soltero & $10,09 \%$ & $24,50 \%$ & $19,42 \%$ & $\mathrm{p}<0,05$ \\
Casado & $79,82 \%$ & $50,50 \%$ & $60,84 \%$ & $\mathrm{p}<0,05$ \\
Divorciado/separado & $7,34 \%$ & $14,50 \%$ & $11,97 \%$ & $\mathrm{p}<0,05$ \\
Viudo & $2,75 \%$ & $10,50 \%$ & $7,77 \%$ & $\mathrm{p}<0,05$ \\
Nivel educacional & & & & \\
Básica & $14,8 \%$ & $33,6 \%$ & $26,9 \%$ & $\mathrm{p}<0,05$ \\
Media & $46,3 \%$ & $36,7 \%$ & $40,1 \%$ & $\mathrm{p}<0,05$ \\
Técnica & $25,9 \%$ & $20,9 \%$ & $22,7 \%$ & $\mathrm{p}<0,05$ \\
Universitaria & $12,9 \%$ & $8,6 \%$ & $10,2 \%$ & $\mathrm{p}<0,05$ \\
Ocupación & & & & \\
Desempleado & $5,45 \%$ & $2,5 \%$ & $3,55 \%$ & $\mathrm{p}<0,05$ \\
Dueña de casa & $0,0 \%$ & $47 \%$ & $30,32 \%$ & $\mathrm{p}<0,05$ \\
Empleado (depend.) & $37,27 \%$ & $19,5 \%$ & $25,81 \%$ & $\mathrm{p}<0,05$ \\
Trab. independiente & $24,55 \%$ & $8 \%$ & $13,87 \%$ & $\mathrm{p}<0,05$ \\
Jubilado & $32,73 \%$ & $23 \%$ & $26,45 \%$ & $\mathrm{p}<0,05$ \\
Factores de riesgo & & & & \\
Diabetes (\%) & $25,7 \%$ & $20 \%$ & $22 \%$ & $\mathrm{NS}$ \\
Tabaquismo (\%) & $22,7 \%$ & $52,5 \%$ & $18,71 \%$ & $\mathrm{NS}$ \\
Colesterol alto (\%) & $39,5 \%$ & $49,5 \%$ & $48 \%$ & $\mathrm{p}<0,05$ \\
Obesidad (\%) & $27,2 \%$ & $54 \%$ & $42,1 \%$ & $\mathrm{p}<0,05$ \\
Sedentarismo (\%) & $33,6 \%$ & & & $\mathrm{p}<0,05$ \\
\hline
\end{tabular}


Tabla 2. Valores de presión arterial y características del tratamiento antihipertensivo en población total y según sexo (información expresada como porcentaje o promedio \pm DE)

\begin{tabular}{|c|c|c|c|c|}
\hline & Hombres (n: 110) & Mujeres (n: 200) & Total (n: 310 ) & $\mathbf{p}$ \\
\hline $\begin{array}{l}\mathrm{N}^{\circ} \text { comp. HTA } \\
1 \\
2 \\
30+\end{array}$ & $\begin{array}{c}2,05 \pm 1,1 \\
37,74 \% \\
36,79 \% \\
25,47 \%\end{array}$ & $\begin{array}{c}2.04 \pm 1,06 \\
33,85 \% \\
44,62 \% \\
21,53 \%\end{array}$ & $\begin{array}{c}2,04 \pm 1,09 \\
35,22 \% \\
41,86 \% \\
22,92 \%\end{array}$ & $\begin{array}{l}\text { NS } \\
\text { NS } \\
\text { NS } \\
\text { NS }\end{array}$ \\
\hline $\begin{array}{l}\text { Prescripción horaria } \\
\text { Matinal } \\
\text { Tarde } \\
\text { Noche }\end{array}$ & $\begin{array}{l}97,27 \% \\
32,04 \% \\
45,79 \%\end{array}$ & $\begin{array}{l}96,95 \% \\
22,99 \% \\
63,54 \%\end{array}$ & $\begin{array}{l}97,07 \% \\
26,21 \% \\
57,19 \%\end{array}$ & $\begin{aligned} & N S \\
< & 0,1 \\
< & 0,05\end{aligned}$ \\
\hline $\begin{array}{l}\text { Antihipertensivos } \\
\text { Inhibidores de ECA } \\
\text { Bloq. canales Ca } \\
\text { Bloq. R. Angiotensina } \\
\text { Diuréticos } \\
\text { Beta-bloqueantes }\end{array}$ & $\begin{array}{l}43 \% \\
11,8 \% \\
41,8 \% \\
24,5 \% \\
8,1 \%\end{array}$ & $\begin{array}{l}34,5 \% \\
10,5 \% \\
51 \% \\
32,5 \% \\
13,5 \%\end{array}$ & $\begin{array}{l}37,74 \% \\
10,97 \% \\
47,74 \% \\
29,68 \% \\
11,61 \%\end{array}$ & $\begin{array}{l}\text { NS } \\
\text { NS } \\
\text { NS } \\
\text { NS } \\
\text { NS }\end{array}$ \\
\hline $\begin{array}{l}\text { Presión arterial } \\
\text { PAS }(\mathrm{mmHg}) \\
\text { PAD }(\mathrm{mm} \mathrm{Hg})\end{array}$ & $\begin{array}{r}145,9 \pm 17,7 \\
82,7 \pm 11,9\end{array}$ & $\begin{aligned} 140,8 & \pm 37 \\
77,9 & \pm 11,8\end{aligned}$ & $\begin{array}{r}142,6 \pm 31,6 \\
79,6 \pm 12,1\end{array}$ & $\begin{array}{l}<0,1 \\
<0,05\end{array}$ \\
\hline
\end{tabular}

\section{Alimentación saludable}

En relación a la alimentación, se observó un SHA promedio de la población de 0,56 $\pm 0,21$. Sólo 16 (5\%) pacientes presentaron un SHA óptimo de 1 . Al analizar la PAS y PAD según el SHA, se observó que a medida que mejora el score mejora la presión arterial sisto-diastólica (Tabla 3); de este modo los pacientes con score 0 presentaron una PAS de $156 \pm 20 \mathrm{mmHg}$ vs score 1 de $137 \pm$ $14 \mathrm{mmHg}$; $y$ PAD de $81 \pm 5 \mathrm{mmHg}$ vs score $1 \mathrm{de}$ $77 \pm 13 \mathrm{mmHg}$.

\section{Adherencia a fármacos antihipertensivos}

La adherencia al TrHTA por MGL fue de 54\%. Analizada la variable adherencia en modelo de regresión logística con: edad, sexo, estado civil, ocupación, nivel educacional, $\mathrm{n}^{\circ}$ de comprimidos, tipo de fármacos, horario de administración, efectos adversos, EQ5D, y relación médico paciente; sólo la edad y la condición de desempleado fueron factores asociados significativamente a adherencia a terapia antihipertensiva (Tabla 4). Analizando la adherencia según quintiles de edad de la población, se observa un porcentaje superior de adherentes en los 2 últimos quintiles, correspondiente a mayores de 64 años (Tabla 5).

En relación a la $\mathrm{PA}$, la $\mathrm{PAD}$ promedio de los
Tabla 3. PAS y PAD según valor de Score de Hábito Alimenticio (SHA) (información expresada como promedio \pm DE)

\begin{tabular}{|rrcc|}
\hline SHA & $\mathbf{n}$ & $\begin{array}{c}\text { PAS } \mathbf{~ m m H g} \\
\text { (DE) }\end{array}$ & $\begin{array}{c}\text { PAD } \mathbf{~ m m H g} \\
\text { (DE) }\end{array}$ \\
\hline 0 & 4 & $156 \pm 20$ & $81 \pm 5$ \\
, 20 & 25 & $145 \pm 23$ & $86 \pm 13$ \\
\hline 40 & 90 & $140 \pm 19$ & $80 \pm 11$ \\
\hline 60 & 112 & $141 \pm 17$ & $80 \pm 12$ \\
\hline 80 & 63 & $142 \pm 21$ & $77 \pm 10$ \\
\hline 1 & 16 & $137 \pm 14$ & $77 \pm 13$ \\
\hline Total & 310 & $141 \pm 19$ & $80 \pm 12$ \\
\hline
\end{tabular}

adherentes vs los no adherentes fue significativamente menor de $78 \pm 12 \mathrm{mmHg}$ vs $81 \pm 17 \mathrm{mmHg}$ $(\mathrm{p}<0,01)$. La PAS promedio de los adherentes vs los no adherentes no objetivó diferencia, siendo de $141 \pm 19 \mathrm{mmHg}$ vs $140 \pm 18 \mathrm{mmHg}$ (NS).

Los pacientes adherentes al TrHTA presentaron un SHA de 0,58 vs el grupo no adherente de 0,54 , lo cual fue significativo $(\mathrm{p}<0,05)$ con un error standard de 0,011 .

De la información obtenida de pacientes que 
Tabla 4. Modelo de regresión logística buscando asociación de factores con adherencia a terapia antihipertensiva

\begin{tabular}{|c|c|c|c|c|}
\hline \multirow[b]{2}{*}{ Edad } & \multicolumn{2}{|c|}{ Modelo 1} & \multicolumn{2}{|c|}{ Modelo 2} \\
\hline & $0,00996^{* *}$ & $(0,00387)$ & $0,00826^{* *}$ & $(0,0033)$ \\
\hline Sexo & 0,127 & $(0,0866)$ & \multicolumn{2}{|c|}{-------- } \\
\hline Estado civil (casado) & $-0,00622$ & $(0,0795)$ & $-0,06664$ & $(0,0669)$ \\
\hline Escolaridad & 0,0124 & $(0,0111)$ & & \\
\hline $\mathrm{N}^{\circ}$ de comprimidos & 0,0433 & $(0,0513)$ & & \\
\hline Horario comprimidos & $-0,385$ & $(0,258)$ & & \\
\hline Clase antihipertensivo IECA & $-0,0652$ & $(0,0784)$ & $-0,08287$ & $(0,0595)$ \\
\hline Autovalencia (EQ5D) & 0,179 & $(0,229)$ & 0,2645 & $(0,174)$ \\
\hline Relación médico paciente & 0,0883 & $(0,0854)$ & 0,06494 & $(0,0647)$ \\
\hline Nivel Educ: Media & & & 0,03808 & $(0,077)$ \\
\hline Nivel Educ: Técnica & & & 0,05867 & $(0,0895)$ \\
\hline Nivel Educ: Universitaria & & & 0,09928 & $(0,1089)$ \\
\hline Ocupación: Trabajador Indep. & & & 0,08571 & $(0,0999)$ \\
\hline Ocupación: Jubilado & & & 0,09505 & $(0,0980)$ \\
\hline Ocupación: Dueña de casa & & & 0,09792 & $(0,0813)$ \\
\hline Ocupación: Desempleado & & & $0,29238^{* *}$ & $(0,1426)$ \\
\hline Clase Medic: Diuréticos & & & $-0,07876$ & $(0,0641)$ \\
\hline Score alimentación (SHA) & & & 0,1781 & $(0,144)$ \\
\hline
\end{tabular}

(Modelos estadísticos indicando efectos marginales de NA a terapia anti HTA) coef beta (error standard) la significancia $* * p<0,05$.

Tabla 5. Porcentaje de adherencia a terapia (Test MGL) por quintiles de edad (expresados en promedio + DE, se adiciona coeficiente de variación

\begin{tabular}{|cccccccc|}
\hline $\begin{array}{c}\text { Quintil de } \\
\text { Edad }\end{array}$ & n & \multicolumn{2}{c|}{ Edad x (DE) } & Coef. Variación & $\begin{array}{c}\text { Test de Morinsky } \\
\text { adherente }\end{array}$ & No Adherente & p \\
\hline 1 & 62 & $44,2(5)$ & 0,114172 & $33,8 \%$ & $66,2 \%$ & $<0,05$ \\
\hline 2 & 62 & $55,1 \quad(2,5)$ & 0,0454716 & $50 \%$ & $50 \%$ & NS \\
\hline 3 & 62 & $61,7(1,4)$ & 0,0230586 & $55,1 \%$ & $41,9 \%$ & $<0,05$ \\
\hline 4 & 62 & $66,8(1,4)$ & 0,0209414 & $66,1 \%$ & $33,9 \%$ & $<0,05$ \\
\hline 5 & 62 & $73,3(3)$ & 0,042081 & $61,3 \%$ & $38,7 \%$ & $<0,05$ \\
\hline Total & 310 & $60,2(10,4)$ &, 1731641 & $54 \%$ & $46 \%$ & $<0,05$ \\
\hline
\end{tabular}

declararon no tomar su prescripción farmacológica en forma regular, la opción más seleccionada para justificarlo fue el olvido en $67 \%$. Otras alternativas fueron efectos indeseados en $10 \%$, despreocupación en $5 \%$, motivos laborales en $4 \%$, y falta de tiempo en $3 \%$.

\section{Autopercepción de salud (EQ5D)}

Se observó autopercepción óptima de salud (bienestar y autovalencia) en $26 \%$ de la muestra. Se detectó menor percepción de salud en mujeres en especial en condiciones de dolor y angustia/ depresión $(\mathrm{p}<0,05)$. El valor promedio de EQ5D 
fue de $0,81 \pm 0,17$ sin diferencia entre adherentes y no adherentes.

\section{Discusión}

En este estudio de hipertensos de CESFAM del Área Metropolitana se detectó 54\% de adherencia al TrHTA por MGL; valor cercano a $48 \%$ detectado por Ingaramo y col. utilizando cuestionario de MGL en Argentina 9 . Esta cifra es mayor a 36\% reportado en Chile, con hipertensos de $\geq 12$ meses de seguimiento en programa cardiovascular ${ }^{14}$, esperable ya que la adherencia farmacológica disminuye a lo largo del tiempo.

Algunos estudios relacionados al cumplimiento de TrHTA no reportan diferencia significativa en cifras tensionales entre adherentes y no adherentes $^{8-14}$, entendible ya que la PA depende de variables difíciles de controlar, tales como la "adecuada" prescripción médica y el factor bata blanca. Al respecto se lograría mayor precisión en el registro de la PA con el monitoreo continuo de PA (MAPA), sin embargo, escasos estudios de adherencia lo han incorporado posiblemente por razones económicas ${ }^{15}$.

En nuestro estudio, los adherentes al TrHTA sí presentaron PAD más baja, sin cambio significativo en PAS. Nuestra explicación radica en la mayor edad de los adherentes $(62,4 \pm 9$ vs $57,7 \pm 11$ años; $\mathrm{p}:<0,01)$ siendo importante recordar que en población adulta mayor de 60 años existe incremento gradual de PAS. Al respecto Brinker y col, en un reciente estudio determinando adherencia por niveles sanguíneos de fármacos, detectaron que los hipertensos adherentes vs los no adherentes eran más viejos y con menor PAD, sin diferencias en $\mathrm{PAS}^{16}$.

El conocer los factores asociados a la adherencia farmacológica antihipertensiva es vital para diseñar políticas públicas. En la literatura internacional existen reportes donde los factores más asociados son: edad, género, estado socioeconómico y número de agentes antihipertensivos. La asociación entre edad y adherencia es compleja. En este estudio, la edad se encontró como factor asociado en forma significativa e independiente a adherencia de fármacos antihipertensivos, observándose mejor adherencia en pacientes mayores de 64 años. Esta observación es consistente con otros estudios $^{6-17-18}$, planteando mayor preocupa- ción del adulto mayor por su condición de salud, independiente de la polifarmacia.

La condición de desempleado sorprendentemente fue otra variable asociada significativamente a mejor adherencia al TrHTA. Esta situación ha sido descrita en población china, sin poder identificar con precisión las razones ${ }^{16}$. En la población chilena estudiada perteneciente a centros de salud existe garantizada por Ley la atención y entrega de fármacos, a diferencia de otros países o comunidades, donde estar desempleado implica pérdida del seguro de salud. Así la condición de estar desempleado sólo le permite al sujeto estar más disponible a asistir a controles médicos y retirar terapia, lo cual paradojalmente resulta beneficioso. Sin embargo, las condiciones de jubilado o dueña de casa no demostraron una asociación significativa.

No encontramos asociación entre género y adherencia, a diferencia de otros que observaron menor adherencia en hombres 9 . En relación a la ausencia de correlación entre el número de comprimidos o agentes antihipertensivos, creemos que se debe al reducido número promedio de comprimidos diarios; ya que existe clara asociación negativa con mayor número de comprimidos $\mathrm{y}$ adherencia farmacológica ${ }^{19}$.

Egan y col. encontraron que el olvido, los eventos adversos y el no desear tomar medicamentos eran las principales razones esgrimidas ante la inadecuada adherencia al TrHTA en población norteamericana ${ }^{20}$. Nos parece destacable que la razón más mencionada por los pacientes de este estudio fue el olvido. Parece fundamental el reforzar el hábito de la toma del fármaco. Al respecto, existe evidencia de que actividades educativas enfocadas en adherencia han sido exitosas en Latinoamérica ${ }^{21}$. A la vez, parece atractivo el apoyo con llamadas telefónicas o mensajería de texto, lo cual ya es una herramienta utilizada a nivel internacional ${ }^{22-23}$.

También es fundamental para el control de la HTA un comportamiento adherente a una dieta saludable con restricción de sal. Esta coherencia al TrHTA en la vida real no se cumple, es así como $80 \%$ de los pacientes fallan con las restricciones alimentarias, ejercicio, disminución del consumo de tabaco y alcohol ${ }^{24}$. En nuestro estudio encontramos un hábito alimentario regular. Las indicaciones nutricionales para el control de la HTA son claras y mandatorias en las guías nacionales. Nuestros resultados traducen 
que a pesar de realizarse consejería nutricional, los pacientes no entienden las indicaciones, o no se sienten motivados para realizar cambios de estilo de vida; lo cual sigue siendo un reto para el equipo médico.

Existe una bien establecida relación entre la ingesta de sodio y PA elevada. Así, el bajar el contenido de sodio en $50 \mathrm{mmol} /$ día $(3 \mathrm{~g}$ sal) de la dieta del hipertenso puede reducir la PAS entre 5-10 $\mathrm{mmHg}^{25-26}$. Esta correlación entre hábito alimentario y PA se observó en este estudio, así los pacientes que manifestaron seguir una dieta más saludable presentaron menor PA. La diferencia de PAS entre los scores extremos fue de $19 \mathrm{mmHg}$, incluso mayor a la esperada por los $5 \mathrm{~g}$ de sal estimados de diferencia. Adicionalmente se observó un valor de SHA significativamente mejor en el grupo adherente por MGL vs el no adherente, así el paciente adherente a la prescripción farmacológica también lo es a la dieta.

Finalmente, las guías chilenas de HTA incorporan el cuestionario de MGL en el algoritmo de evaluación del paciente con falta de control de la PA, por ser una herramienta accesible, sin embargo, carecemos de información local en relación a la sensibilidad y especificidad. Es importante plantear la utilidad de confrontar los resultados de MGL con otros métodos de cuantificación de adherencia, tales como contabilización de droga o sistema electrónico de monitoreo. Sea cualquiera el método utilizado, la evidencia apunta a que la población hipertensa más adherente presenta menor tasa de eventos cardiovasculares ${ }^{27}$.

Dentro de las limitaciones del estudio destacamos no haber contado con MAPA para determinación de PA con mayor precisión, el cual sólo se considera en el programa $\mathrm{CV}$ ante condiciones especiales como HTA refractaria. Otras limitaciones fueron no haber determinado sodio urinario como marcador de restricción salina, y la determinación de adherencia sólo por autorreporte. Por otro lado, las principales fortalezas son ser un estudio multicéntrico, realizado por equipo independiente a los CESFAM, que incorporó comunas de distinto nivel socioeconómico.

En conclusión, la mitad de los hipertensos del programa cardiovascular no son adherentes al TrHTA a 6 meses de prescripción a nivel de CESFAM del Área Metropolitana. Es todo un desafío encontrar herramientas que permitan un mejor cumplimiento.
Un Anexo que informa la estimación de consumo de sal por SHA está a disposición de los lectores que lo soliciten al autor corresponsal.

\section{Referencias}

1. Kearney P, Whelton M, Reynolds K, Munter P, Whelton P, He J. Global burden of hypertension: analysis of worldwide data. Lancet 2005; 365: 217-23.

2. Lawes CM, Vander Hoorn S, Rodgers A, for the International Society of Hypertension. Global burden of blood-pressure-related disease, 2001. Lancet 2008; 371: 1513-8.

3. MINSAL. Encuesta Nacional 2009-2010. http://www. redsalud.gov.cl/portal/url/item/99 Cl2b89738d80d5e04001011e0113f8.pdf

4. Sackett DL. The hypertensive patient: 5. Compliance with therapy. CMA Journal, 1979; 121: 259-61.

5. Jones JK, Gorkin L. A study of United Kingdom population. Br Med J 1995; 311: 293-5.

6. Monane M, Bohn R, Gurwitz, Glynn R, Levin R, Avon J. Compliance with Antihypertensive Therapy among Elderly Medicaid Enrollees: The Roles of Age, Gender and Race. Am J Public Health 1996; 86: 1805-8.

7. Lars Osterberg L, Blaschke T. Adherence to Medication. N Engl J Med 2005; 353: 487-97.

8. Morinsky DE, Green LW, Levine DM. Concurrent and predictive validity of self-reported mesure of medication compliance. Med Care 1986; 24: 67-74.

9. Ingaramo R, Vita N, Bendersky M, Arnolt M, Bellido C, Piskorz D, et al. Estudio Nacional Sobre Adherencia al Tratamiento (ENSAT) Rev Fed Arg Cardiol 2005; 34: 104-11.

10. Lopes N, Zanini A, Casella-Filho A, Palandri A. Metabolic Syndrome patient compliance with drug treatment Clinics 2008; 63 (5): 573-80.

11. Guía Clínica de Hipertensión Arterial Primaria o Esencial en personas de 15 años o más, Ministerio de Salud de Chile. 2010.

12. Herdman M, Badia X, Berra S. El EQ-5D: una alternativa sencilla para la medición de la calidad de vida relacionada con la salud en atención primaria. Atención Primaria 2001; 28: 425-9.

13. Mattes RD, Donnelly D. Relative contributions of dietary sodium sources. J Am Coll Nutr 1991; 10: 383-93.

14. Garrido J, Chacón J, Sandoval D, Muñoz R, López N, Oyarzún E, et al. Control del Hipertenso, Avances logrados en Chile mediante el Programa de Salud Cardiovascular. Rev Chil Cardiol 2013; 32: 85-96.

15. Nuesch R, Schroeder K, Dieterle T, Martina B, Battegay 
E. Relation between insufficient response to antihypertensive treatment and poor compliance with treatment: a prospective case control study. BMJ 2001; 323: 142-6.

16. Brinker S, Pandey A, Ayers C, Price A, Raheja P, Arbique $D$, et al. Therapeutic Drug Monitoring Facilitates Blood Pressure Control in Resistant Hypertension. J Am Coll Cardiol 2014; 63: 834-5.

17. Hashmi S, Afridi M, Abbas K, Sajwani R, Saleheen D, Frossard $\mathrm{P}$, et al. Factors associated with adherence to anti-hypertensive treatment in Pakistan. PLoS ONE 2007; 2 (3): e280.

18. Lee G, Wang H, Liu K, Cheung Y, Morinsky D, Wong M. Determinants of medication adherence to antithypertensive medications among a Chinese population using Morinsky Medication Adherence Scale. Plos ONE 2013: 8 (4): e 62775.

19. Iskedjian M, Einarson T, MacKeigan L, Shear N, Addis A, Mittmann N, et al. Relationship between daily dose frequency and adherence to antihypertensive pharmacotherapy: evidence from a meta-analysis. Clinical Therapeutics 2002; 24: 302-16.

20. Egan BH, Lackland DT, Cutler NE. Awareness, knowledge and attitudes of older Americans about high blood pressure. Arch Intern Med 2003; 163: 681-7.

21. Márquez E, Casado J, Celotti B, Gascon J, Martin de Pablos J, Gil R, et al. El cumplimiento terapéutico en la hipertensión arterial. Ensayo sobre la intervención durante 2 años mediante educación sanitaria. Aten Primaria 2000; 26: 5-10.

22. Friedman R, Kazis L, Jette A, Smith M, Stollerman J, Torgerson J, et al. A telecommunications system for monitoring and counseling patients with hypertension: Impact on medication adherence and blood pressure control. American Journal of Hypertension 1996; 9: 285-92.

23. Free C, Knight R, Robertson S, Whittaker R, Edwards P, Zhou W, et al. Smoking cessation support delivered via mobile phone text messaging (txt2stop): a single-blind, randomised trial. Lancet 2011; 378: 49-55,

24. DiMatteo R, Giordani P, Lepper H, Croghan T. Patient adherence and medical treatment outcomes: a meta-analysis. Medical Care 2002; 40: 794-811.

25. Law M, Frost C, Wald N. Analysis of data from trials of salt redution BMJ 1991; 302: 819-24.

26. Cutler J, Follam D, Allendes P. Randomized trial of sodium reduciton: an overviw. An J Clin Nutr 1997; 65 (suppl): 643S-651S.

27. Mazzaglia G, Ambrosioni E, Alacqua M, Filippi A, Sessa $\mathrm{E}$, Inmordino $\mathrm{V}$, et al. Adherence to antihipertensive medications and cardiovascular morbidity among newly diagnosed Hypertensive patientes. Circulation 2009; 120: 1598-605. 